\title{
La similitude des barrages mobiles - Expériences de l'Usine de la Gentille
}

\author{
par MM. C. Camichel, L. Escande et E. Crausse
}

Dans nos recherches antéricures, nous avons étudié, à diverses reprises, la similitude des barrages-déversoirs fonctionnant avec ou sans contractions latérales (1). Nous avons comparé, en par-

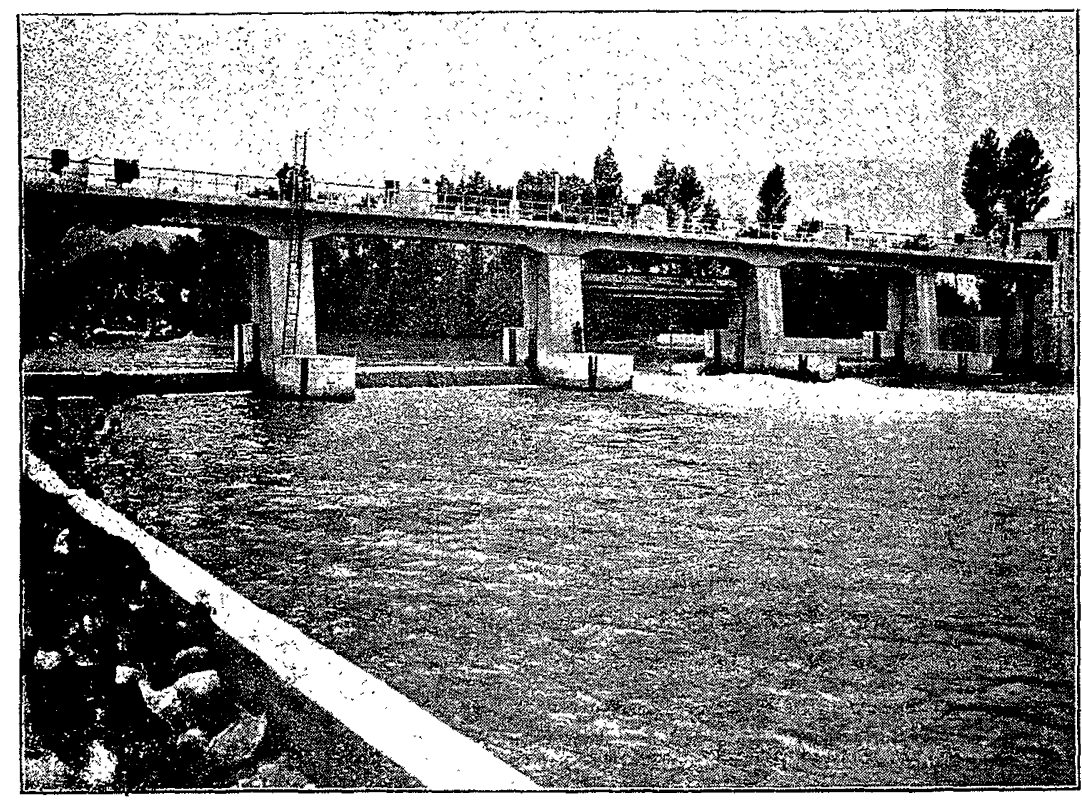

Fig. 1

ticulier, une série de modèles reproduisant un même ouvrage, différentes échelles, variant de $1 / 300$ à $1 / 10$, entre modẻles extrêmes; nous avons constaté que la loi de similitude de ReechFroude s'applique, avec une approximation très grande, à la comparaison des débits qui s'écoulent sur les divers modèles, tant que l'échelle n'est pas trop réduite; nous avons également montré que les modèles très petits de barrages sans contractions latérales fournissent, pour une charge donnée, des valeurs du débit relativement trop faibles, par suite de l'importance plus grande de la couche limite au contact du parement. Au contraire, dans le cas où il existe des contractions latérales notables, cellesci sont d'autant plus réduites que l'échelle est plus petite, de telle sorte-que leur influence compense largement l'effet de la couche limite et rend relativement trop fort le débit des très petits modèles.

Dans ces recherches antérieures, toutefois, nous n'avions jamais eu l'occasion d'effectuer une comfraraison directe entre un ouvrage hydraulique indu triel et ses modèles réduits; de plus ,nous 'n'avions pas étudié le cas des barrages noyés, dont le débit est sous la dépendance du niveau aval.

Nous avons pu compléter nos recherches, par l'étude de ces deux points, en effectuant simultanément des

(1) Comptes-rendus, t. 180, 1925, p. $99-186,1928$, p. 28 $-187,1928$, p. $766-16^{4}$, 1929, p. $317-194,1932$, p. 807. mesures sur la passe centrale du barrage à vanne mobile de la Gentille, établi à Saint-Gaudens, sur la Garonne, par la Société des Forces Motrices du Comminges, (fig. 1) et sur un modèle réduit de cet ouvrage, à l'échelle $1 / 10$, installé dans le canal d'essais du laboratoire de Banlève.

Nous avons choisi cet ouvrage de la Gentille, en raison des conditions particulièrement favorables qu'il présente poutr l'exécution des essais : en effet, dans les conditions de notre expérience, niveau amont à la cote 345,53 , niveau aval à la cote 344,58 , la cote du seuil étant 343,85 , le débit qui s'écoule et que nous avons mesuré en explorant une section droite avec des moulinets, est égal à $46,53 \mathrm{~m}^{3} / \mathrm{sec}$. Cette valeur du débit correspond au régime normal de la Garonne dans la période actuelle en tenant compte des débits dérivés de l'usine. L'expérience peut donc être effectuée en dehors d'une période de crue, ce qui évite les inconvénients que peuvent présenter, pour les appareils de mesure, les corps flottants ou les blocs entraînés par l'eau dans ces périodes. De plus, la différence de niveau entre l'amont et l'aval, est assez faible pour que les vitesses ne dépassent guère les limites atteintes dans les opérations de tarage des moulinets; enfin, l'existence d'un batardeau susceptible d'effectuer, par commande électrique, des translations suivant une horizontale ou une verticale, dans le plan d'une section droite, donne toute facilité pour l'exploration de celle-ci à l'aide des moulinets. Les fig. 2 et 3 représentent la passe étudiée, sur le barrage réel et sur lè modèle réduit, les photogra. phies étant prises de l'extrémité amont de la pile constituant la rive gauche de cette passe. On retrouve sur le modèle réduit toutes les grandes lignes caractérisant l'écoulement sur le bar-

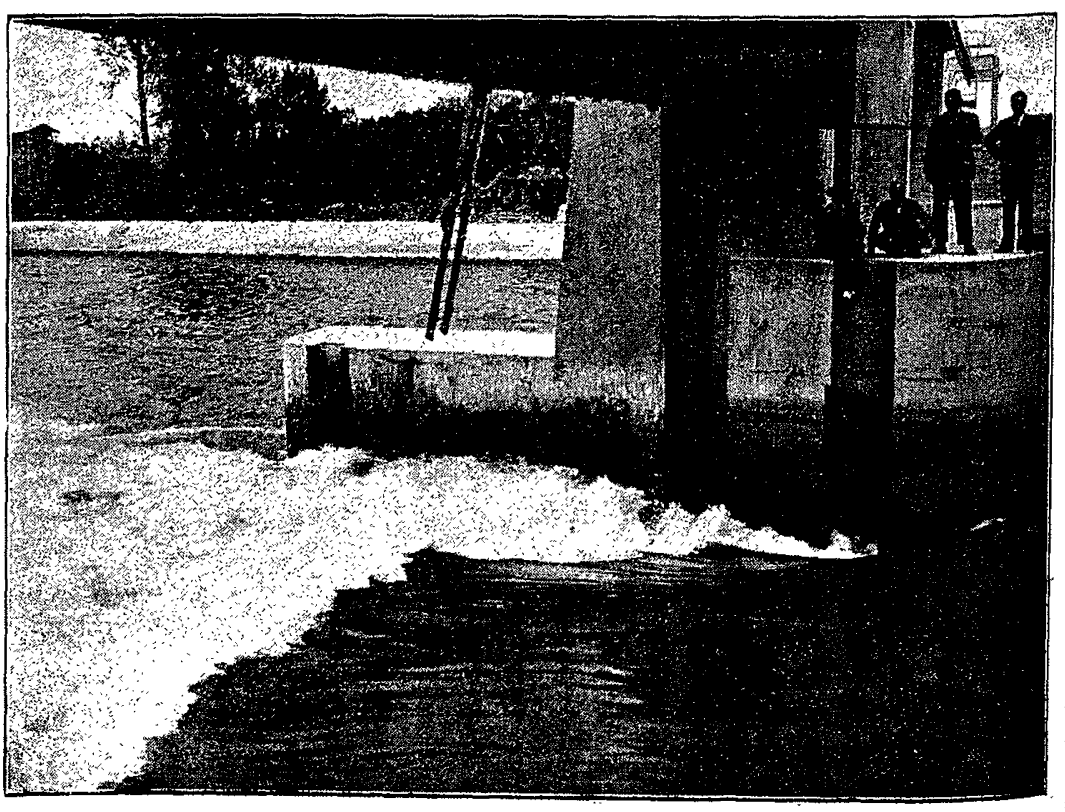

Fig. 2 
rage; contraction initiale, puis intumescence, enlin abaissement de la surface libre vers le niveau aval; une seule différence l'aspect se remarque immédiatement, c'est l'entraînement d'air créant dans l'ouvrage un déferlemenl superficiel qui n'existe pas dans le modèle; c'est d'ailleurs là un phénomène tıes général dans les études sur les modèles réduits. re pratiquement exacle dans la comparaison du barrage el du modèle au $1 / 10$.

Nous avons également déterminé (cet élément nous était d'ailleurs nécessaire pour la mesure du débit du barrage), le profil en travers de la surface libre de l'eau, dans la section droite correspondant aux mesures faites au moulinet, et dans la section

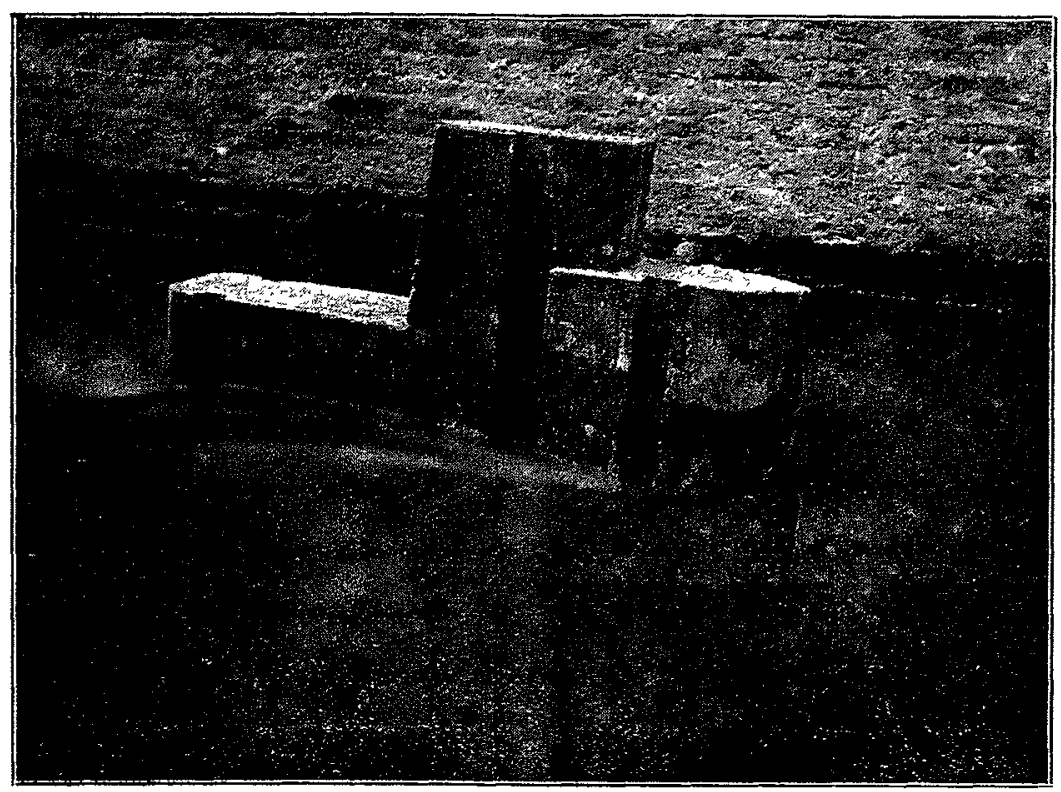

Fig. 3

L'expérience de similitude a été réalisée de la manière suivanle: nous avons fait passer, à travers le modèle réduit, le débit homologue de celui de $46,53 \mathrm{~m}^{3} / \mathrm{sec}$. mesuré à la Gentille, soit $147,31 / \mathrm{sec}$. et nous avons réglé le plan d'eau à l'aval du modèle, à une cole correspondant à une profondeur homologue de celle qui existait à l'aval du barrage réel ; nous avons alors mesuré la cote du plan homologue du modèle; la fig. 4 montre la différence très nette entre ces deux profils ; conformément à ce qui est indiqué plus haut, la contraction latérale est beaucoup plus forte dans le barrage que dans le modèle, la valeur de la section mouillée n'étant d'ailleurs pas sensiblement modifiée; la forme du profil en travers accuse donc des divergences notables vis à vis de la similitude.

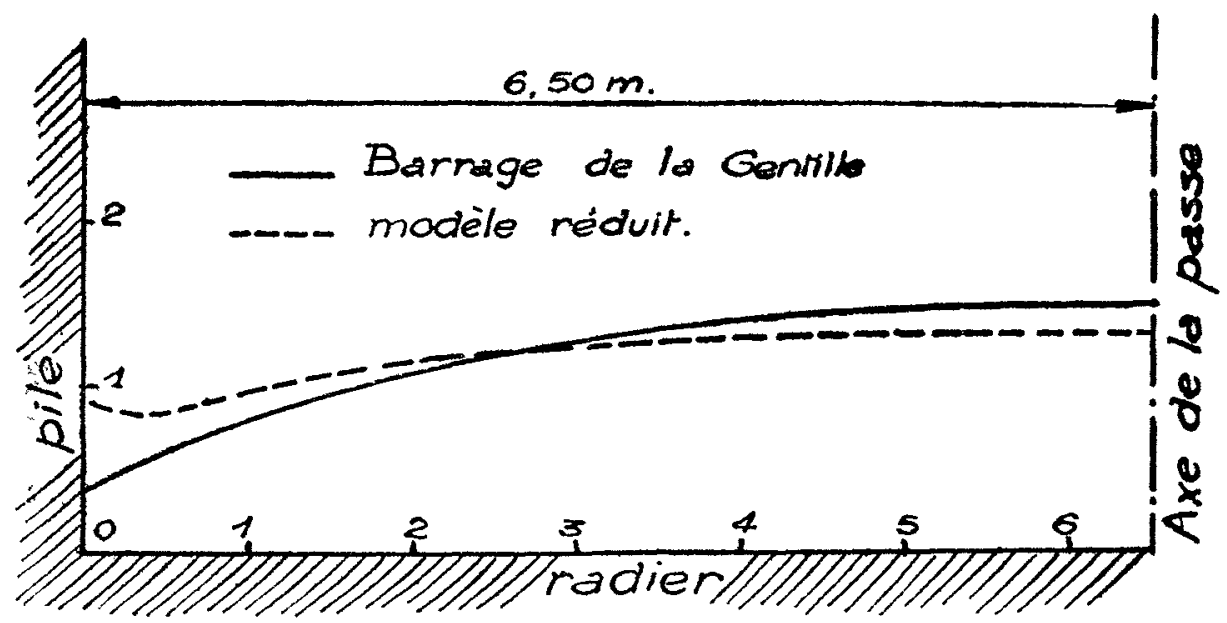

Fig. 4

d'eau en amont du modèle et constaté que celle-ci correspondail à une hauteur au dessus du seuil de $16,75 \mathrm{~cm}$. ; le chiffre correspondant, résultant de la cote du plan d'eau en amont du barrage réel, ramené à l'échelle du modèle, est de $16,8 \mathrm{~cm}$., d'après les cotes données ci-dessus pour le niveau amont et pour le seuil ; la concordance est donc excellente et l'on voit que la similitude des débits, sous des charges homologues, est vérifiée d'une maniè-
On voit donc que ces expériences, venant achever la série d'éţudes que nous avons entreprises sur la similitude de l'écoulement dans les barrages, si elles soulignent la divergence déjà indiquée de la forme des profils en travers dans le cas de contractions latérales, permettent de conclure d'une manière définitive à l'exactitude de la détermination globale du débit de ces ouvrages par des études sur modèles réduits. 